\title{
Prediction of Rock Mechanical Properties of Shale Gas Reservoir based on Relevant Finite Element Models
}

\author{
Ni Weijun \\ Key Laboratory of MOE for Petroleum Engineering,China University of Petroleum (Beijing); Beijing, China \\ College of Petroleum Engineering, Xi'an Shiyou University, Xi'an Shaanxi, China
}

Li Qi

Key Laboratory of MOE for Petroleum Engineering,China University of Petroleum (Beijing); Beijing, China College of Petroleum Engineering, Xi'an Shiyou University, Xi'an Shaanxi, China

Li Xumei

Petroleum Technology Research Institute of Petrochina Changqing Oilfield Company, Xi'an Shaanxi, China

\section{Zhou Tingting}

Research Institute,Petro China Coalbed Methane Linfen branch, Taiyuan Shanxi ,China

\begin{abstract}
At present, many scholars have researched and analyzed laws generated by micro-annulus and proposed relevant finite element models and analytical models. But the existing models fail to consider the effects of stratum environment and apply stress fields and more parameters to the models in research of micro-annulus, which is unable to accurately describe mechanical effects of cementing interface in the construction process. In our research, we have proposed a new model which is based on fractional step finite element method. stress change of assemblies in the process of drilling, cementing and fracturing in construction has been considered and a casing pipe- cement sheath- stratum finite element model based on the anisotropy of shale stratum and pore percolation has been set up. The model is able to have a more realistic simulation on production regularity of cementing interface's micro-annulus in shale stratum so as to further certain theoretical directions on construction operations on the site.
\end{abstract}

Keywords- Prediction, rock mechanical properties, shale gas reservoir, Intelligent Algorithm

\section{INTRODUCTION}

Shale gas reservoir has the characteristics of low porosity and extremely low matrix permeability and it is increasingly produced by fracturing transformation. At present, the staged fracturing technology of horizontal well and hydraulic sand is mostly adopted for the development of shale gas in Sichuan, China. In staged fracturing transformation process, the problem of wellbore integrity occurs in many wells, which means damage and distortion of casing pipes in follow-up fracturing transformation process have caused that fracturing tools are unable to down, bridge plug can't be drilled after completion of transformation and production log cannot be carried on later, and seriously affected the smooth progress of the development of shale gas [1-3]. 
According to the mechanism that the load of casing pipe is larger than its yield stress resulting in the damage of casing pipes, some scholars have carried out study about the influence of non-uniform stress load and those are caused by cementing on the strength of casing pipe and the influences laws of uneven load on cement's shear stress and tensile stress under the condition of different eccentricity ratios. [4]

\section{THEORETICAL MODEL FOR ANISOTROPIC ELASTIC DEFORMATION OF STRATIFICATION SHALE IN SHALE}

Shale reservoir is subject to natural fracture and its bedding configuration has directivity. The elastic mechanics parameters have obvious anisotropy especially when they are parallel and vertical to bedding surface. The Thesis has considered the characteristic for transverse isotropy of stratification shale. Stratification stratum is the simplest form of anisotropic formation in which shale is generally regarded as a model in engineering. The characteristic of stress-strain of shale in bedding surface is isotropic and it can be described by 5 independent elastic constants. Hooke Law reflects the relationship between stress and strain in elastomers. For anisotropic materials, its constitutive equation of stress-strain can be expressed as follows:

$$
\begin{aligned}
\left\{\varepsilon^{\prime}\right\}= & D^{\prime-1}\left\{\sigma^{\prime}\right\} \\
D^{\prime-1}= & {\left[\begin{array}{cccccc}
\frac{1}{E_{x}} & -\frac{v_{x y}}{E_{y}} & -\frac{\nu_{x z}}{E_{z}} & & \\
-\frac{v_{x y}}{E_{y}} & \frac{1}{E_{y}} & -\frac{\nu_{y z}}{E_{z}} & & \\
-\frac{\nu_{x z}}{E_{z}} & -\frac{v_{y z}}{E_{z}} & \frac{1}{E_{z}} & \frac{1}{G_{y z}} & & \\
& & & & \frac{1}{G_{x z}} & \\
& & & & & \frac{1}{G_{x y}}
\end{array}\right] }
\end{aligned}
$$

Where, $\mathrm{Ex}=\mathrm{Ez}$ is a elasticity modulus parallel to isotropic planes; Ey is a elasticity modulus vertical to isotropic planes;

Vxz is a poisson ratio parallel to isotropic planes; $\mathrm{Vyz}=\mathrm{Vxy}$ is a poisson ratio vertical to isotropic planes.

Shear modulus in isotropic planes $\mathrm{XOZ}$ is:

$$
G_{x z}=G_{h}=\frac{E_{h}}{2\left(1+v_{h}\right)}
$$

Where, Gh is a shear modulus parallel to isotropic planes; GPa;E is a elasticity modulus vertical to isotropic planes; vh is a poisson ratio parallel to isotropic planes.

Batugin and other scholars have proposed mathematic solutions of the fifth elastic constant $\mathrm{Gv}=\mathrm{Gyz}=\mathrm{Gxy}$ which are vertical to isotropy's YOZ plane and XOY plane and they have verified the solution through abundant experimental data [5]

$$
G_{V}=G_{y z}=G_{x y}=\frac{E_{y} E_{z}}{E_{y}+E_{z}+2 v_{y z} E_{z}}
$$




\section{FUNDAMENTAL THEORY OF FLUID-STRUCTURE INTERACTION}

Terzaghi consolidation theory considers that total stress distribution of soil mass in solidification process is unchanged, but the assumption is limited to 3D models because it ignores the process of stress loading. Biot gave a "True Three-dimensional Consolidation Theory” from a comparatively strict consolidation mechanism, which truly reflected the relationship between altering pore pressure and rock mass deformation. Suppose that the rock is with macroscopic isotropy, linear elasticity, small deformation and seepage flow isotropy and it meets Darcy Law, the following is Biot consolidation equation expressed by displacement and pore pressure [6]:

$$
\left\{\begin{array}{c}
-G \nabla^{2} u^{s}-\frac{G}{1-2 v}-\frac{\partial}{\partial x}\left(\frac{\partial u^{s}}{\partial x}+\frac{\partial v^{s}}{\partial y}+\frac{\partial w^{s}}{\partial z}\right)+\frac{\partial u}{\partial x}=0 \\
-G \nabla^{2} v^{s}-\frac{G}{1-2 v}-\frac{\partial}{\partial y}\left(\frac{\partial u^{s}}{\partial x}+\frac{\partial v^{s}}{\partial y}+\frac{\partial w^{s}}{\partial z}\right)+\frac{\partial u}{\partial y}=0 \\
-G \nabla^{2} w^{s}-\frac{G}{1-2 v}-\frac{\partial}{\partial z}\left(\frac{\partial u^{s}}{\partial x}+\frac{\partial v^{s}}{\partial y}+\frac{\partial w^{s}}{\partial z}\right)+\frac{\partial u}{\partial z}=0 \\
-\frac{\partial}{\partial t}\left(\frac{\partial u^{s}}{\partial x}+\frac{\partial v^{s}}{\partial y}+\frac{\partial w^{s}}{\partial z}\right)+\frac{k}{\gamma_{w}} \nabla^{2} u=0
\end{array}\right.
$$

Where $\nabla^{2=}\left(\frac{\partial^{2}}{\partial x}+\frac{\partial^{2}}{\partial y}+\frac{\partial^{2}}{\partial z}\right)$.

Where: $\mathrm{G}$ is a shear modulus; $\mathrm{V}$ is Poisson's ratio of solid particles; $\mathrm{u}$ is pole fluid stress; $\gamma_{w}$ is a volume weight of fluid; $\mathrm{k}$ is an osmotic coefficient of solid particles; $\mathrm{w}$ is overall displacement; $\nabla^{2=}$ is a laplace operator. In these equations, the first three are consolidation equations and the fourth is fluid continuity equation. Three unknowns $u^{s}$, $v^{s}$ and $w^{s}$ contained in the equations can be solved on certain initial conditions and boundary conditions.

\section{ESTABLISHMENT OF FINITE ELEMENT MODEL}

Establishment of finite element's local coordinate system and sensitivity parameters determination.

In view of transverse isotropy characteristic of stratification shale, a local coordinate system () of material is set up after the establishment of numerical models. Suppose the world coordinate in the built stratum model is XYZ, isotropic plane is $\mathrm{X}-\mathrm{Z}$, axis of symmetry is $\mathrm{Y}$ and the horizontal well is drilled along the orientation of minimum horizontal principal stress. Local coordinate system of the bedding surface on shale is $X^{\prime} Y^{\prime} Z^{\prime}$ ' with three-axis in one-to-one correspondence with global coordinate system so as to be convenient for the setting of boundary conditions. The

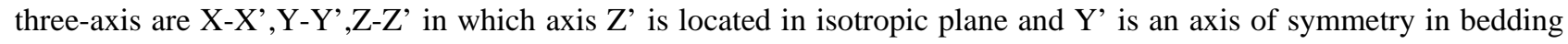
surface. 


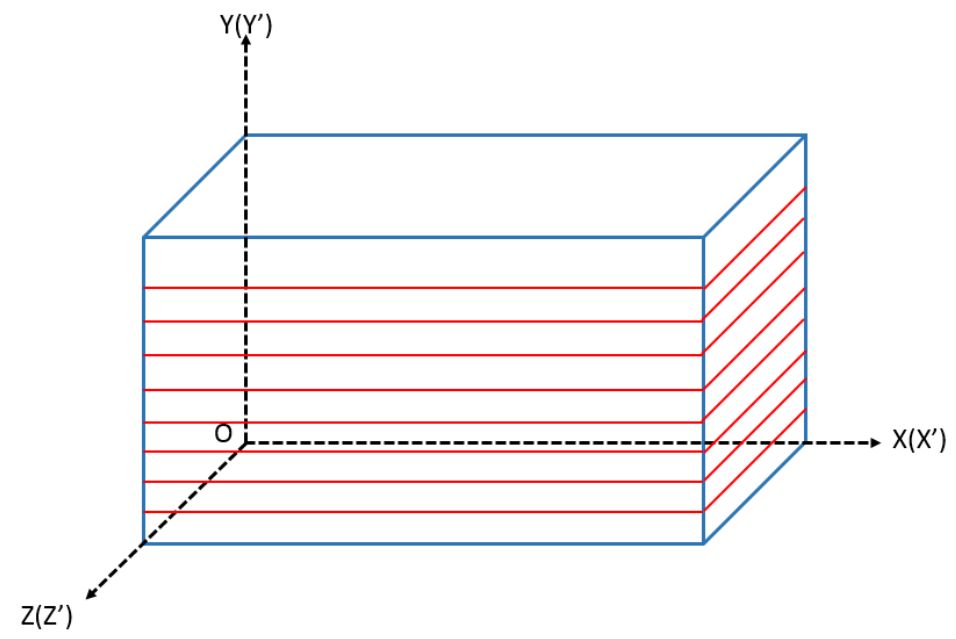

Fig 1. Global Coordinate System and Local Coordinate System of Stratification Shale

\section{Model establishment}

The Thesis adopts finite element software to directly couple temperature field and stress field of assembly. Casing pipe-cement sheath-stratum assembly in the model undergoes the external load from far-field stress and inside of casing pipes together. To reflect the situations of stratum response more truthful and avoid the deviation from model sizes, the simulation uses pre-defined function in finite element software to upload initial ground stress and initial temperature for assembly and constraint the outer boundary fully. The method of heat transfer in assembly is called transient heat transfer.

1) Rock heat transfer, which is vertical to the direction of borehole axis, is a only access to heat conduction in assembly.

2) Thermophysics in assembly (density, specific heat and pyroconductivity) are not affected by temperature.

3) Casing pipe, cement sheath and stratum are isotropic materials.

4) Assemblies are contacted closely with no slideness.

Detailed geometrical parameters, material features and thermodynamic parameters in the assembly model are shown in Table 1 and 2.

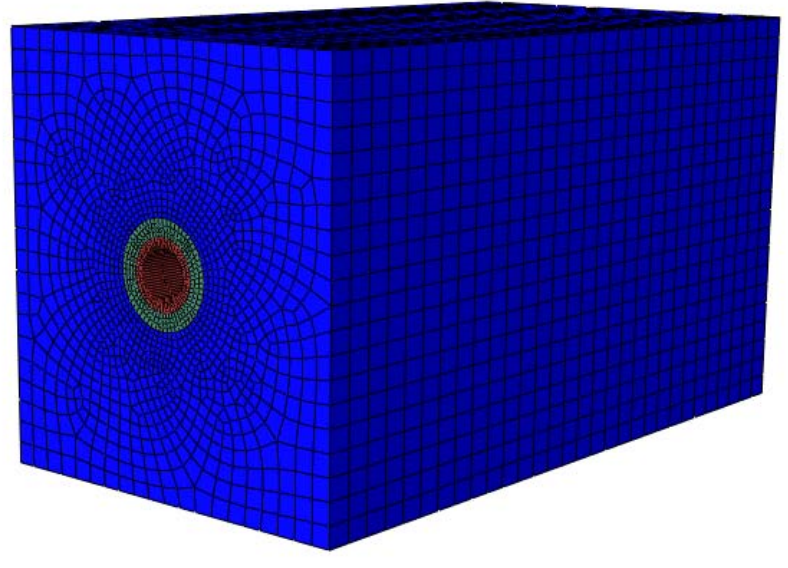

Fig 2. The Combination Finite Model of Casing Pipe-Cement Sheath-Formation

Table-1 Geometrical Parameters and Material Characteristics on Casing Pipe-Cement Sheath

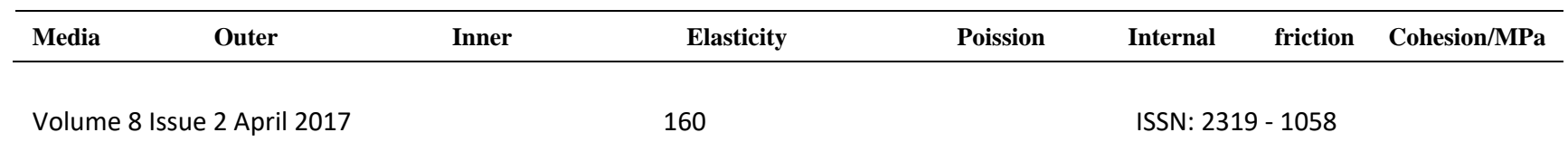




\begin{tabular}{cccccc}
\hline & diameter/mm & diameter/mm & modulus/GPa & ratio & angle/ ${ }^{\circ}$ \\
\hline Casing pipe & 139.7 & 120.3 & 210 & 0.3 & - \\
Cement & 215.9 & 139.7 & 9 & 0.15 & 21.6 \\
sheath & & & & & \\
\hline
\end{tabular}

Table-2 Thermodynamic Parameters on Model Material

\begin{tabular}{ccccc}
\hline Media & Density $/\left(\mathrm{kg} \cdot \mathrm{m}^{-3}\right)$ & Expansion parameter $/{ }^{\circ} \mathrm{C}^{-1}$ & Specific heat $/\left(\mathrm{J} \cdot \mathrm{kg}^{-1} \cdot{ }^{0} \mathrm{C}^{-1}\right)$ & Heat conductivity $/\left(\mathrm{W} \cdot \mathrm{m}^{-1} \cdot{ }^{0} \mathrm{C}^{-1}\right)$ \\
\hline Casing pipe & 7800 & $1.22 \mathrm{e}-5$ & 460 & 45 \\
Cement sheath & 1800 & $1.05 \mathrm{e}-5$ & 865 & 0.9 \\
Stratum & 2300 & $1.03 \mathrm{e}-5$ & 896 & 2.2 \\
\hline
\end{tabular}

In construction process of shale gas well fracturing, large displacement of high pump pressure will be adopted to conduct reservoir alteration. Constant injecting of fluids will result in dynamic changes on well's temperature field, and assembly load in transient heat transfer process will be changed over time. Therefore, model parameters in the Thesis set the initial ground temperature as $100 \mathrm{C}$, fluid te mperature as $0^{\circ} \mathrm{C}$ and the duration of fluid-injection is $1 \mathrm{~h}$. Where, initial hydrostatic pressure is 10MPa in casing pipes and shaft pressure is 90MPa in fluid-injection. According to the fact that shale gases' horizontal well track is generally parallel to the orientation of minimum paralleling principal stress, the boundary load of the model can be set as: horizontal direction expresses maximum paralleling principal stress and vertical direction expresses vertical principal stress with the size of $\sigma \mathrm{V}=30 \mathrm{MPa}$ and $\sigma \mathrm{H}=\sigma \mathrm{h} 20 \mathrm{MPa}$ respectively. Rock elasticity modulus: $\mathrm{Ey}=27 \mathrm{GPa} ; \mathrm{EX}=\mathrm{EZ}=27 \sim 35 \mathrm{GPa}$; rock poisson ratio: $\mathrm{vy}=0.223$; $\mathrm{vx}=\mathrm{vZ}=0.242$; stratum saturation: $100 \%$; porosity: $3 \%$; fluid density; $0.98 \mathrm{~g} / \mathrm{cm} 3$, permeability: 1.0 e-3um2 and pore pressure: 5MPa.

\section{CONCLUSION}

Transient heat transfer model of anisotropic shaft assembly based on sub-step finite element method can be used to better evaluate the laws of micro-annulus generation; when ignoring the effect of temperature, micro-annulus is generated in the first interface in which surrounding-wall stress and the size of micro-annulus is distributed unevenly. Anisotropy of mechanical parameters in stratum has a small effect on the size of micro-annulus in which the decreasing ground stress in fracturing process and increasing formation pore pressure will result in the increasing micro-annulus accordingly.

\section{ACKNOWLEDGEMENT}

National Science Foundation of China (51574194), Project of Special Science Study of Shaanxi Education Department (16JK1613、15JK1567) ,Project of science and technology industry of Shaanxi Province (2014K05-02) , Youth Science and Technology Innovation Funded by xi’an shiyou university（2010QN011、2016BS09）.

\section{REFERENCES}

[1] Li Y, Jiang Y, Zhao J, "Extended finite element method for analysis of multi-scale flow in fractured shale gas reservoirs,Environmental Earth Sciences", vol. 73, pp. 6035-6045,2015.

[2] Sheng M, Li G, Huang Z, "Hydro-mechanical coupling model of shale gas reservoir and its finite element analysis", Yanshilixue Yu Gongcheng Xuebao/chinese Journal of Rock Mechanics \& Engineering, vol. 32, pp. 1894-1900,2013.

[3] Doyle H, Lohfeld S, Mcdonnell P, " Evaluation of a Multiscale Modelling Methodology to Predict the Mechanical Properties of PCL/ß-TCP Sintered Scaffold Materials", Annals of Biomedical Engineering, vol. 43, pp.1-10, 2015.

[4] Nie X, Zou C, Li Z, "Numerical simulation of the electrical properties of shale gas reservoir rock based on digital core", Journal of 
Geophysics and Engineering, vol. 13, pp.481-490,2016.

[5] Räss L, Yarushina V M, Simon N S C, " Chimneys, channels, pathway flow or water conducting features - an explanation from numerical modelling and implications for CO 2, storage ", Energy Procedia, vol. 63, pp.3761-3774,2014.

[6] Cook J, Frederiksen R A, Hasbo K, "Rocks Matter: Ground Truth in Geomechanics", Oilfield Review, vol. 19, pp.36-55,2007. 